Vol. 70, No. 1 (2018) pp. 387-402

doi: $10.2969 / \mathrm{jmsj} / 07017538$

\title{
Topology of mixed hypersurfaces of cyclic type
}

\author{
By Kazumasa Inaba, Masayuki Kawashima and Mutsuo Oka
}

(Received May 31, 2016)

\begin{abstract}
Let $f_{I I}(\boldsymbol{z}, \overline{\boldsymbol{z}})=z_{1}^{a_{1}+b_{1}} \bar{z}_{1}^{b_{1}} z_{2}+\cdots+z_{n-1}^{a_{n-1}+b_{n-1}} \bar{z}_{n-1}^{b_{n-1}} z_{n}+$ $z_{n}^{a_{n}+b_{n}} \bar{z}_{n}^{b_{n}} z_{1}$ be a mixed weighted homogeneous polynomial of cyclic type and $g_{I I}(\boldsymbol{z})=z_{1}^{a_{1}} z_{2}+\cdots+z_{n-1}^{a_{n-1}} z_{n}+z_{n}^{a_{n}} z_{1}$ be the associated weighted homogeneous polynomial where $a_{j} \geq 1$ and $b_{j} \geq 0$ for $j=1, \ldots, n$. We show that two links $S_{\varepsilon}^{2 n-1} \cap f_{I I}^{-1}(0)$ and $S_{\varepsilon}^{2 n-1} \cap g_{I I}^{-1}(0)$ are diffeomorphic and their Milnor fibrations are isomorphic.
\end{abstract}

\section{Introduction.}

Let $f(\boldsymbol{z}, \overline{\boldsymbol{z}})$ be a mixed polynomial of complex variables $\boldsymbol{z}=\left(z_{1}, \ldots, z_{n}\right)$ given as

$$
f(\boldsymbol{z}, \overline{\boldsymbol{z}}):=\sum_{i=1}^{m} c_{i} \boldsymbol{z}^{\nu_{i}} \overline{\boldsymbol{z}}^{\mu_{i}},
$$

where $c_{i} \in \mathbb{C}^{*}$ and $\boldsymbol{z}^{\nu_{i}}=z_{1}^{\nu_{i, 1}} \ldots z_{n}^{\nu_{i, n}}$ for $\nu_{i}=\left(\nu_{i, 1}, \ldots, \nu_{i, n}\right)$ (respectively $\overline{\boldsymbol{z}}^{\mu_{i}}=$ $\bar{z}_{1}^{\mu_{i, 1}} \ldots \bar{z}_{n}^{\mu_{i, n}}$ for $\left.\mu_{i}=\left(\mu_{i, 1}, \ldots, \mu_{i, n}\right)\right)$. Here $\bar{z}_{j}$ represents the complex conjugate of $z_{j}$.

A point $\boldsymbol{w} \in \mathbb{C}^{n}$ is called a mixed singular point of $f(\boldsymbol{z}, \overline{\boldsymbol{z}})$ if the gradient vectors of $\mathfrak{R} f$ and $\mathfrak{I} f$ are linearly dependent at $\boldsymbol{w}$. Certain restricted classes of mixed polynomials of the variables $\boldsymbol{z}$ which admit Milnor fibrations had been considered by Seade, see for instance $[\mathbf{7}],[\mathbf{8}]$. The last author introduced the notion of the Newton boundary and the concept of non-degeneracy for a mixed polynomial and he showed the existence of Milnor fibration for the class of strongly non-degenerate mixed polynomials [3].

We consider the classes of mixed polynomials which was first introduced by RuasSeade-Verjovsky [6] and Cisneros-Molina [1]. Let $p_{1}, \ldots, p_{n}$ and $q_{1}, \ldots, q_{n}$ be integers such that $\operatorname{gcd}\left(p_{1}, \ldots, p_{n}\right)=\operatorname{gcd}\left(q_{1}, \ldots, q_{n}\right)=1$. We define the $S^{1}$-action and the $\mathbb{R}^{*}$ action on $\mathbb{C}^{n}$ as follows:

$$
\begin{array}{ll}
c \circ \boldsymbol{z}=\left(c^{p_{1}} z_{1}, \ldots, c^{p_{n}} z_{n}\right), & c \in S^{1}, \\
r \circ \boldsymbol{z}=\left(r^{q_{1}} z_{1}, \ldots, r^{q_{n}} z_{n}\right), & r \in \mathbb{R}^{*} .
\end{array}
$$

If there exists a positive integer $d_{p}$ such that $f(\boldsymbol{z}, \overline{\boldsymbol{z}})$ satisfies

$$
f\left(c^{p_{1}} z_{1}, \ldots, c^{p_{n}} z_{n}, \bar{c}^{p_{1}} \bar{z}_{1}, \ldots, \bar{c}^{p_{1}} \bar{z}_{n}\right)=c^{d_{p}} f(\boldsymbol{z}, \overline{\boldsymbol{z}}), \quad c \in S^{1},
$$

we say that $f(\boldsymbol{z}, \overline{\boldsymbol{z}})$ is a polar weighted homogeneous polynomial. Similarly $f(\boldsymbol{z}, \overline{\boldsymbol{z}})$ is called a radial weighted homogeneous polynomial if there exists a positive integer $d_{r}$ such

2010 Mathematics Subject Classification. Primary 14J17; Secondary 14J70, 58K05.

Key Words and Phrases. polar weighted homogeneous, Milnor fibration. 
that

$$
f\left(r^{q_{1}} z_{1}, \ldots, r^{q_{n}} z_{n}, r^{q_{1}} \bar{z}_{1}, \ldots, r^{q_{n}} \bar{z}_{n}\right)=r^{d_{r}} f(\boldsymbol{z}, \overline{\boldsymbol{z}}), \quad r \in \mathbb{R}^{*} .
$$

Let $f$ be a polar and radial weighted homogeneous polynomial. Then $f$ admits the global Milnor fibration $f: \mathbb{C}^{n} \backslash f^{-1}(0) \rightarrow \mathbb{C}^{*}$, see for instance [6], [1] , [2], [3].

Let $f(\boldsymbol{z}, \overline{\boldsymbol{z}})=\sum_{i=1}^{m} c_{i} \boldsymbol{z}^{\nu_{i}} \overline{\boldsymbol{z}}^{\mu_{i}}$ be a mixed polynomial with $c_{j} \neq 0, j=1, \ldots, m$. Put

$$
g(\boldsymbol{z}):=\sum_{i=1}^{m} c_{i} \boldsymbol{z}^{\nu_{i}-\mu_{i}} .
$$

We call $g$ the associated Laurent polynomial of $f$. A mixed polynomial $f$ is called simplicial if $m \leq n$ and the ranks of the matrices $N \pm M$ are $m$ where $N=\left(\nu_{1}, \ldots, \nu_{n}\right)$ and $M=\left(\mu_{1}, \ldots, \mu_{n}\right)$. Here $\nu_{i}$ and $\mu_{i}$ are considered as column vectors $\nu_{i}=$ ${ }^{t}\left(\nu_{i 1}, \ldots, \nu_{i n}\right), \mu_{i}={ }^{t}\left(\mu_{i 1}, \ldots, \mu_{i n}\right) . \quad f$ is called full if $m=n$. A full simplicial mixed polynomial $f$ and its associated Laurent polynomial $g$ admit a unique polar weight and a unique radial weight in the above sense [2]. It is useful to consider a graph $\Gamma$ associated to $f$. First we associate a vertex $v_{i}$ if $z_{i}$ or $\bar{z}_{i}$ appears in $f$. We join $v_{i}$ and $v_{j}$ by an edge if there is a monomial $\boldsymbol{z}^{\nu_{k}} \overline{\boldsymbol{z}}^{\mu_{k}}$ which contains both variables $z_{i}, z_{j}$. That is $\nu_{k, a}+\mu_{k, a}>0$ for $a=i, j$. Most important graphs are a bamboo graph

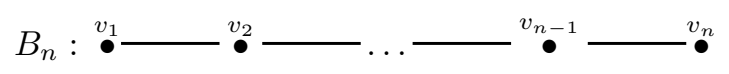

and a cyclic graph $C_{n}$ which is obtained from $B_{n}$ adding an edge between $v_{n}$ and $v_{1}$.

We restrict the Milnor fibrations defined by $f$ and $g$ on the complex torus $\mathbb{C}^{* n}$ where $\mathbb{C}^{* n}=\left(\mathbb{C}^{*}\right)^{n}$. In $[\mathbf{2}$, Theorem 10], it is shown that there exists a canonical diffeomorphism $\varphi: \mathbb{C}^{* n} \rightarrow \mathbb{C}^{* n}$ which gives an isomorphism of the Milnor fibrations defined by $f$ and $g$ :

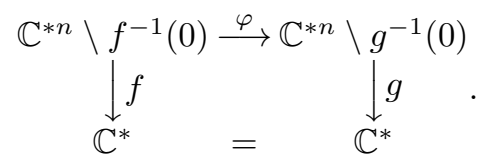

However the canonical diffeomorphism $\varphi$ does not extend to $\mathbb{C}^{n} \backslash\{O\}$ in general. Here $O$ is the origin of $\mathbb{C}^{n}$. The exceptional case is a mixed Brieskorn polynomial, for which this canonical diffeomorphism extends as a continuous homeomorphism [6]. In [4], the last author studied the following simplicial polar weighted homogeneous polynomials:

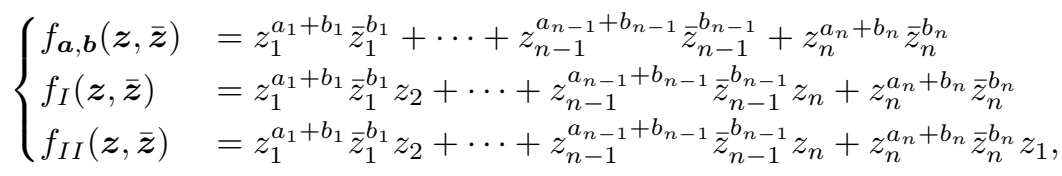

where $a_{j} \geq 1$ and $b_{j} \geq 0$ for $j=1, \ldots, n$. Here the notation is the same as in [4]. Note that the graph of $f_{I}$ is a bamboo and that of $f_{I I}$ is a cyclic graph. The graph of $f_{\boldsymbol{a}, \boldsymbol{b}}$ is $n$ disjoint vertices without any edges. A polar weighted homogeneous polynomial $f_{\boldsymbol{a}, \boldsymbol{b}}(\boldsymbol{z}, \overline{\boldsymbol{z}})$ and a weighted homogeneous polynomial $g_{\boldsymbol{a}}(\boldsymbol{z})$ are called a mixed Brieskorn polynomial and a Brieskorn polynomial respectively. A mixed polynomial $f_{I}(\boldsymbol{z}, \overline{\boldsymbol{z}})$ is 
called a simplicial mixed polynomial of bamboo type and $f_{I I}(\boldsymbol{z}, \overline{\boldsymbol{z}})$ is called a simplicial mixed polynomial of cyclic type respectively. He showed that two links of $f_{\iota}$ and the associated polynomial $g_{\iota}(\boldsymbol{z})$ in a small sphere are isotopic and their Milnor fibrations are isomorphic for $\iota=(\boldsymbol{a}, \boldsymbol{b})$ and $I$. He conjectured the assertion will be also true for the case $f_{I I}$.

\section{Statement of the result.}

The purpose of this paper is to give a positive answer to the above conjecture. Thus we study the following simplicial polynomial $f_{I I}(\boldsymbol{z}, \overline{\boldsymbol{z}})$ and its associated weighted homogeneous polynomial $g_{I I}(\boldsymbol{z})$ :

$$
\left\{\begin{array}{l}
f_{I I}(\boldsymbol{z}, \overline{\boldsymbol{z}})=z_{1}^{a_{1}+b_{1}} \bar{z}_{1}^{b_{1}} z_{2}+\cdots+z_{n-1}^{a_{n-1}+b_{n-1}} \bar{z}_{n-1}^{b_{n-1}} z_{n}+z_{n}^{a_{n}+b_{n}} \bar{z}_{n}^{b_{n}} z_{1}, \\
g_{I I}(\boldsymbol{z})=z_{1}^{a_{1}} z_{2}+\cdots+z_{n-1}^{a_{n-1}} z_{n}+z_{n}^{a_{n}} z_{1}
\end{array}\right.
$$

where $a_{j} \geq 1$ and $b_{j} \geq 0$ for $j=1, \ldots, n$. We assume that $f_{I I}$ contains a conjugate $\bar{z}_{j}$ for some $j$. This implies

(a) there exists $j \in\{1, \ldots, n\}$ such that $b_{j} \geq 1$.

We also assume that $f_{I I}$ is simplicial. As the determinant of $N-M$ is given by $a_{1} \cdots a_{n}+(-1)^{n+1}$, we assume also that

(b) there exists $k \in\{1, \ldots, n\}$ such that $a_{k} \geq 2$.

Though this assumption is not necessary if $n$ is odd, we assume (b) anyway. Since $f_{I I}(\boldsymbol{z}, \overline{\boldsymbol{z}})$ is a polar and radial weighted homogeneous, $f_{I I}(\boldsymbol{z}, \overline{\boldsymbol{z}})$ admits a global fibration

$$
f_{I I}: \mathbb{C}^{n} \backslash f_{I I}^{-1}(0) \rightarrow \mathbb{C}^{*}
$$

$[\mathbf{6}],[\mathbf{1}],[\mathbf{2}],[\mathbf{3}]$. The complex polynomial $g_{I I}(\boldsymbol{z})$ is a weighted homogeneous polynomial with respect to the same polar weight of $f_{I I}$ and $g_{I I}$ with $n=3$ is listed in the classification of weighted homogeneous surfaces in $\mathbb{C}^{3}$ with isolated singularity [5]. We consider the hypersurfaces

$$
V_{f}:=f_{I I}^{-1}(0), \quad V_{g}:=g_{I I}^{-1}(0)
$$

and respective links

$$
K_{f, \varepsilon}=V_{f} \cap S_{\varepsilon}^{2 n-1}, \quad K_{g, \varepsilon}=V_{g} \cap S_{\varepsilon}^{2 n-1}
$$

where $S_{\varepsilon}^{2 n-1}$ is the $(2 n-1)$-dimensional sphere centered at the origin $O$ with radius $\varepsilon$. Then the two links $K_{f, \varepsilon}$ and $K_{g, \varepsilon}$ are smooth for any $\varepsilon>0([\mathbf{2}])$. We consider the following family of mixed polynomials: 


$$
\begin{aligned}
f_{I I, t}(\boldsymbol{z}, \overline{\boldsymbol{z}}):= & (1-t) f_{I I}(\boldsymbol{z}, \overline{\boldsymbol{z}})+t g_{I I}(\boldsymbol{z}) \\
= & (1-t)\left(z_{1}^{a_{1}+b_{1}} \bar{z}_{1}^{b_{1}} z_{2}+\cdots+z_{n-1}^{a_{n-1}+b_{n-1}} \bar{z}_{n-1}^{b_{n-1}} z_{n}+z_{n}^{a_{n}+b_{n}} \bar{z}_{n}^{b_{n}} z_{1}\right) \\
& +t\left(z_{1}^{a_{1}} z_{2}+\cdots+z_{n-1}^{a_{n-1}} z_{n}+z_{n}^{a_{n}} z_{1}\right) \\
= & \sum_{j=1}^{n} z_{j}^{a_{j}} z_{j+1}\left\{(1-t)\left|z_{j}\right|^{2 b_{j}}+t\right\}
\end{aligned}
$$

where $0 \leq t \leq 1$. Here the numbering is modulo $n$, so $z_{n+1}=z_{1}$. Though the mixed polynomial $f_{I I, t}$ is not radial weighted homogeneous for $t \neq 0,1, f_{I I, t}$ is polar weighted homogeneous for $0 \leq t \leq 1$ with the same weight $P=\left(p_{1}, \ldots, p_{n}\right)$ which is characterized by $a_{j} p_{j}+p_{j+1}=d_{p}, j=1, \ldots, n$. Put

$$
V_{t}=f_{I I, t}^{-1}(0), K_{t, \varepsilon}=S_{\varepsilon}^{2 n-1} \cap V_{t}, \quad 0 \leq t \leq 1 .
$$

Note that

$$
\begin{aligned}
& f_{I I, 0}=f_{I I}, f_{I I, 1}=g_{I I} \\
& V_{f}=V_{0}, K_{f, \varepsilon}=K_{0, \varepsilon}, V_{g}=V_{1}, K_{g, \varepsilon}=K_{1, \varepsilon} .
\end{aligned}
$$

First recall that $V_{t}$ has an isolated mixed singularity at the origin $O$ and $V_{t} \backslash\{O\}$ is non-singular for any $0 \leq t \leq 1$ by [4, Lemma 9$]$. Our main result is:

Transversality Theorem 1. Let $V_{t}$ be as above. For any fixed $\varepsilon>0$, the sphere $S_{\varepsilon}^{2 n-1}$ and the family of hypersurfaces $V_{t}$ are transversal for $0 \leq t \leq 1$.

\section{Proof of Transversality Theorem 1.}

\subsection{Strategy of the proof.}

We follow the recipe of $[\mathbf{4}]$. First recall that

$$
\begin{aligned}
f_{I I, t}(\boldsymbol{z}, \overline{\boldsymbol{z}}) & :=(1-t) f_{I I}(\boldsymbol{z}, \overline{\boldsymbol{z}})+t g_{I I}(\boldsymbol{z}) \\
& =\sum_{j=1}^{n} z_{j}^{a_{j}} z_{j+1}\left\{(1-t)\left|z_{j}\right|^{2 b_{j}}+t\right\} .
\end{aligned}
$$

Recall that $V_{t}$ is non-singular off the origin by [4]. To show the transversality of the sphere $S_{\varepsilon_{0}}^{2 n-1}$ and $V_{t}$, we have to show that the Jacobian matrix of $\mathfrak{R} f_{I I, t}, \mathfrak{I} f_{I I, t}$ and $\rho(\boldsymbol{z})$ has rank 3 at every intersection $\boldsymbol{w} \in S_{\varepsilon_{0}}^{2 n-1} \cap V_{t}$. Here $\rho(\boldsymbol{z})=\|\boldsymbol{z}\|^{2}$, the square of the radius $\|\boldsymbol{z}\|$. However this computation is extremely complicated. Instead, we follow the recipe of [4]. We will show the existence of a tangent vector $\boldsymbol{v} \in T_{\boldsymbol{w}} V_{t}$ which is not tangent to the sphere $S_{\varepsilon_{0}}^{2 n-1}$.

Take a point $\boldsymbol{w}=\left(w_{1}, \ldots, w_{n}\right) \in V_{t} \cap S_{\varepsilon_{0}}^{2 n-1}$ and fix it hereafter. To find such a vector $\boldsymbol{v}$, we will construct a real analytic path

$$
\boldsymbol{w}(s)=\left(r_{1}(s) w_{1}, \ldots, r_{n}(s) w_{n}\right)
$$

on a neighborhood of $s=0$ so that $\boldsymbol{w}(0)=\boldsymbol{w}$ and 


$$
f_{I I, t}(\boldsymbol{w}(s), \overline{\boldsymbol{w}}(s))=(s+1) f_{I I, t}(\boldsymbol{w}, \overline{\boldsymbol{w}})
$$

where $r_{j}(s), j=1, \ldots, n$ are real-valued functions on $|s| \ll 1$ which satisfy certain functional equalities. The equality (1) implies that the curve $\boldsymbol{w}(s)$ is an embedded curve in $V_{t}$ with $\boldsymbol{w}(0)=\boldsymbol{w}$. Then we define the vector as the tangent vector of this curve at $s=0$ :

$$
\boldsymbol{v}=\frac{d \boldsymbol{w}}{d s}(0)
$$

To find such a path $\boldsymbol{w}(s)=\left(r_{1}(s) w_{1}, \ldots, r_{n}(s) w_{n}\right)$, we solve a certain functional equation, using the inverse mapping theorem.

\subsection{Construction of $\boldsymbol{w}(s)$.}

First, for $\boldsymbol{w} \in V_{t}$ with $\boldsymbol{w} \neq O$, we consider the following map:

$$
\begin{aligned}
\Phi_{\boldsymbol{w}} & : \mathbb{R}^{n+1} \rightarrow \mathbb{R}^{n+1} \\
\left(r_{1}, \ldots, r_{n}, s\right) & \mapsto\left(h_{1}, \ldots, h_{n}, s\right),
\end{aligned}
$$

where $h_{j}$ is a polynomial function of variables $r_{1}, \ldots, r_{n}$ and $s$ defined by

$$
h_{j}=r_{j}^{a_{j}} r_{j+1}\left\{(1-t)\left|w_{j}\right|^{2 b_{j}} r_{j}^{2 b_{j}}+t\right\}-(s+1)\left\{(1-t)\left|w_{j}\right|^{2 b_{j}}+t\right\}, j=1, \ldots, n
$$

where $t$ is fixed on $0 \leq t \leq 1$. Here the numbering is modulo $n$, so $r_{n+1}=r_{1}$. We want to solve the equations $h_{1}=\cdots=h_{n}=0$ in $r_{1}, \ldots, r_{n}$ expressing $r_{j}$ as a function of $s$ so that we get the system of equations

$$
h_{j}\left(r_{1}(s), \ldots, r_{n}(s), s\right) \equiv 0, j=1, \ldots, n .
$$

This equality is equivalent to (1) which is more explicitly written as

$$
\begin{aligned}
f_{I I, t}(\boldsymbol{w}(s), \overline{\boldsymbol{w}}(s)) & =(s+1) f_{I I, t}(\boldsymbol{w}, \overline{\boldsymbol{w}}) \quad \text { where } \\
f_{I I, t}(\boldsymbol{w}(s), \overline{\boldsymbol{w}}(s)) & =\sum_{j=1}^{n}\left(r_{j}(s) w_{j}\right)^{a_{j}}\left(r_{j+1}(s) w_{j+1}\right)\left\{(1-t)\left|w_{j}\right|^{2 b_{j}} r_{j}(s)^{2 b_{j}}+t\right\}, \\
(s+1) f_{I I, t}(\boldsymbol{w}, \overline{\boldsymbol{w}}) & =(s+1) \sum_{j=1}^{n} w_{j}^{a_{j}} w_{j+1}\left\{(1-t)\left|w_{j}\right|^{2 b_{j}}+t\right\} .
\end{aligned}
$$

We will solve the functional equality (1) using the inverse mapping theorem.

Lemma 1. Let $\boldsymbol{w} \in V_{t}$ with $\boldsymbol{w} \neq O$ and $0<t<1$. Then the Jacobian matrix $J\left(\Phi_{\boldsymbol{w}}\right)$ has rank $n+1$ at $\left(r_{1}, \ldots, r_{n}, s\right)=(1, \ldots, 1,0)$, where 


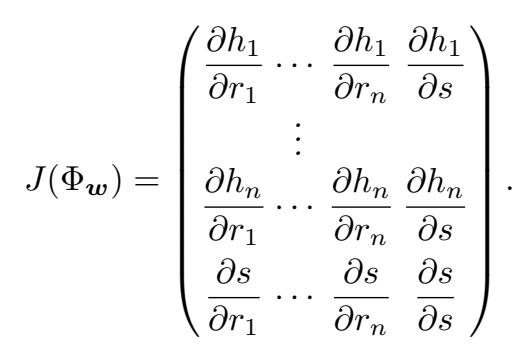

Proof. By a direct computation, the Jacobian matrix of $\Phi_{\boldsymbol{w}}$ is given as

$$
J\left(\Phi_{\boldsymbol{w}}\right)=\left(\begin{array}{cccccc}
\alpha_{1,1} & \alpha_{1,2} & 0 & \ldots & 0 & -\beta_{1} \\
0 & \alpha_{2,2} & \alpha_{2,3} & \ddots & \vdots & \vdots \\
\vdots & \ddots & \ddots & \ddots & \vdots & \vdots \\
0 & \ldots & 0 & \alpha_{n-1, n-1} & \alpha_{n-1, n} & \vdots \\
\alpha_{n, 1} & 0 & \ldots & 0 & \alpha_{n, n} & -\beta_{n} \\
0 & \ldots & \ldots & \ldots & 0 & 1
\end{array}\right),
$$

where

$$
\begin{aligned}
\alpha_{j, j} & =r_{j}^{a_{j}-1} r_{j+1}\left\{(1-t)\left|w_{j}\right|^{2 b_{j}}\left(a_{j}+2 b_{j}\right) r_{j}^{2 b_{j}}+a_{j} t\right\}, \\
\alpha_{j, j+1} & =r_{j}^{a_{j}}\left\{(1-t)\left|w_{j}\right|^{2 b_{j}} r_{j}^{2 b_{j}}+t\right\}, \\
\beta_{j} & =\left\{(1-t)\left|w_{j}\right|^{2 b_{j}}+t\right\}, \quad j=1, \ldots, n .
\end{aligned}
$$

Since $0<t<1, \alpha_{j, j}$ and $\alpha_{j, j+1}$ at $(1, \ldots, 1,0)$ are positive real numbers for each $j=1, \ldots, n$. If $\alpha_{j, j}$ and $\alpha_{j, j+1}$ are not evaluated at $(1, \ldots, 1,0)$, they may be negative, for instance $\alpha_{j, j}$ is negative if $r_{j}$ is positive and $r_{j+1}$ is negative. The determinant $\operatorname{det} J\left(\Phi_{\boldsymbol{w}}\right)$ is given as

$$
\begin{aligned}
\operatorname{det} J\left(\Phi_{\boldsymbol{w}}\right)= & \alpha_{1,1} \ldots \alpha_{n, n}+(-1)^{n+1} \alpha_{1,2} \cdots \alpha_{n-1, n} \alpha_{n, 1} \\
= & \prod_{j=1}^{n} r_{j}^{a_{j-1}} r_{j+1}\left\{(1-t)\left|w_{j}\right|^{2 b_{j}}\left(a_{j}+2 b_{j}\right) r_{j}^{2 b_{j}}+a_{j} t\right\} \\
& +(-1)^{n+1} \prod_{j=1}^{n} r_{j}^{a_{j}}\left\{(1-t)\left|w_{j}\right|^{2 b_{j}} r_{j}^{2 b_{j}}+t\right\} .
\end{aligned}
$$

The proof of Lemma 1 is reduced to the following assertion.

Assertion 1. $\operatorname{det} J\left(\Phi_{\boldsymbol{w}}\right)>0$.

Proof. (i) If $n$ is an odd number, $\operatorname{det} J\left(\Phi_{\boldsymbol{w}}\right)$ at $(1, \ldots, 1,0)$ is obviously positive. (ii) Suppose that $n$ is a positive even number. Consider

$$
\alpha_{j, j}^{\prime}:=r_{j}^{a_{j}}\left\{(1-t)\left|w_{j}\right|^{2 b_{j}}\left(a_{j}+2 b_{j}\right) r_{j}^{2 b_{j}}+a_{j} t\right\} .
$$

Note that $\prod_{j=1}^{n} \alpha_{j, j}=\prod_{j=1}^{n} \alpha_{j, j}^{\prime}$. We have the following. 


$$
\begin{aligned}
& \operatorname{det} J\left(\Phi_{\boldsymbol{w}}\right)=\prod_{j=1}^{n} \alpha_{j, j}-\prod_{j=1}^{n} \alpha_{j, j+1}=\prod_{j=1}^{n} \alpha_{j, j}^{\prime}-\prod_{j=1}^{n} \alpha_{j, j+1} \\
& \operatorname{det} J\left(\Phi_{\boldsymbol{w}}\right) \geq 0 \Longleftrightarrow \prod_{j=1}^{n} \frac{\alpha_{j, j}^{\prime}}{\alpha_{j, j+1}} \geq 1 .
\end{aligned}
$$

As $\alpha_{j, j}^{\prime} \geq \alpha_{j, j+1}$, the equality takes place if $\alpha_{j, j}^{\prime}=\alpha_{j, j+1}$ for $j=1, \ldots, n$. We assume that $\alpha_{j, j}^{\prime}=\alpha_{j, j+1}$ at $(1, \ldots, 1,0)$ for any $j, 1 \leq j \leq n$. Then

$$
(1-t)\left|w_{j}\right|^{2 b_{j}}\left(a_{j}+2 b_{j}\right)+a_{j} t=(1-t)\left|w_{j}\right|^{2 b_{j}}+t, j=1, \ldots, n
$$

and this is the case if and only if $\left(w_{j}, a_{j}\right)=(0,1)$ or $\left(a_{j}, b_{j}\right)=(1,0)$. Thus the Jacobian of $\Phi_{\boldsymbol{w}}$ at $(1, \ldots, 1,0)$ is equal to 0 if and only if $\left(w_{j}, a_{j}\right)=(0,1)$ or $\left(a_{j}, b_{j}\right)=(1,0)$ for $j=1, \ldots, n$. However these cases do not happen, since by assumption (b) there exists $j$ with $a_{j} \geq 2$. Thus the assertion is proved. This completes also the proof of Lemma 1.

Now we are ready to prove the transversality of $S_{\varepsilon_{0}}^{2 n-1}$ and $V_{t}$ for any $\varepsilon_{0}>0$ and $0 \leq t \leq 1$

\subsection{Proof of Transversality Theorem.}

The assertion is known for $t=0,1$ by [3]. Thus we assume that $0<t<1$. Recall that $f_{I I, t}: \mathbb{C}^{n} \rightarrow \mathbb{C}$ has a unique singularity at the origin $O$ for any $0 \leq t \leq 1$ by $[\mathbf{4}$, Lemma 9]. As the codimension of $T_{\boldsymbol{w}} S_{\varepsilon_{0}}^{2 n-1}$ in $\mathbb{C}^{n} \cong \mathbb{R}^{2 n}$ is 1 , to show the transversality, it suffices to show the existence of a vector $\boldsymbol{v} \in T_{\boldsymbol{w}} V_{t}$ with $\boldsymbol{v} \notin T_{\boldsymbol{w}} S_{\varepsilon_{0}}^{2 n-1}$.

For a given $\boldsymbol{w}=\left(w_{1}, \ldots, w_{n}\right) \in V_{t}$, we consider the nullity set $I_{\boldsymbol{w}}=\left\{i \mid w_{i}=0\right\}$.

Case 1: $I_{\boldsymbol{w}}=\emptyset$. This is the most essential case and does not appear for the mixed polynomials $f_{\boldsymbol{a}, \boldsymbol{b}}$ and $f_{I}$. The corresponding graph is cyclic.

By Lemma 1 , the Jacobian of $\Phi_{\boldsymbol{w}}$ at $(1, \ldots, 1,0)$ is non-zero. By the Inverse mapping theorem, there exist a neighborhood $U \subset \mathbb{R}^{n+1}$ of $(1, \ldots, 1,0)$ and a neighborhood $W \subset$ $\mathbb{R}^{n+1}$ of $\Phi_{\boldsymbol{w}}(1, \ldots, 1,0)=(0, \ldots, 0)$ and a real analytic mapping $\Psi_{\boldsymbol{w}}=\left(\psi_{1}, \ldots, \psi_{n}, \mathrm{id}\right)$ : $\mathrm{W} \rightarrow \mathrm{U}$ so that

$$
\Phi_{\boldsymbol{w}} \circ \Psi_{\boldsymbol{w}}=\mathrm{id}_{\mathrm{W}} \text { and } \Psi_{\boldsymbol{w}} \circ \Phi_{\boldsymbol{w}}=\mathrm{id}_{\mathrm{U}} .
$$

Put $0:=(0, \ldots, 0) \in \mathbb{R}^{n}$ and consider $V \subset \mathbb{R}:=W \cap(\{\mathbf{0}\} \times \mathbb{R})$, a neighborhood of $0 \in \mathbb{R}$ and define smooth functions $r_{j}: V \rightarrow \mathbb{R}$ of the variable $s$ by $r_{j}(s):=\psi_{j}(0, \ldots, 0, s)$. Note that $r_{j}(0)=1$. We have the equalities:

$$
h_{j}\left(r_{1}(s), \ldots, r_{n}(s), s\right) \equiv 0, s \in V, j=1, \ldots, n .
$$

As we have seen in the above discussion, this implies

$$
r_{j}^{a_{j}}(s) r_{j+1}(s)\left\{(1-t)\left|w_{j}\right|^{2 b_{j}} r_{j}(s)^{2 b_{j}}+t\right\}-(s+1)\left\{(1-t)\left|w_{j}\right|^{2 b_{j}}+t\right\} \equiv 0,
$$

which implies 


$$
\begin{aligned}
f_{I I, t}(\boldsymbol{w}(s), \overline{\boldsymbol{w}}(s)) & =\sum_{j=1}^{n} r_{j}(s)^{a_{j}} r_{j+1}(s) w_{j}^{a_{j}} w_{j+1}\left\{(1-t)\left|w_{j}\right|^{2 b_{j}} r_{j}(s)^{2 b_{j}}+t\right\} \\
& =(s+1) f_{I I, t}(\boldsymbol{w}, \overline{\boldsymbol{w}}) .
\end{aligned}
$$

Thus $f_{I I, t}(\boldsymbol{w}(s), \overline{\boldsymbol{w}}(s)) \equiv 0$. Put $\boldsymbol{v}=d \boldsymbol{w} / d s(0)$. We have $\boldsymbol{v} \in T_{\boldsymbol{w}} V_{t}$ by the definition. Now to finish the proof of the transversality assertion, we need only to show

Assertion 2. $\quad \boldsymbol{v} \neq \mathbf{0}$ and $\boldsymbol{v} \notin T_{\boldsymbol{w}} S_{\varepsilon_{0}}^{2 n-1}$.

To prove the assertion, we consider the differential in $s$ of

$$
h_{j}\left(r_{1}(s), \ldots, r_{n}(s), s\right)=r_{j}(s)^{a_{j}} r_{j+1}(s)\left\{(1-t)\left|w_{j}\right|^{2 b_{j}} r_{j}(s)^{2 b_{j}}+t\right\}-(s+1)\left\{(1-t)\left|w_{j}\right|^{2 b_{j}}+t\right\} .
$$

By a direct computation, we get the equality

$$
\begin{aligned}
\frac{d}{d s} h_{j}\left(r_{1}(s), \ldots, r_{n}(s), s\right) & =\left(\sum_{k=1}^{n} \frac{\partial h_{j}}{\partial r_{k}} \frac{d r_{k}}{d s}\right)-\beta_{j} \\
& =\alpha_{j, j} \frac{d r_{j}}{d s}+\alpha_{j, j+1} \frac{d r_{j+1}}{d s}-\beta_{j} \equiv 0
\end{aligned}
$$

where

$$
\begin{aligned}
\alpha_{j, j} & =r_{j}^{a_{j}-1} r_{j+1}\left\{(1-t)\left|w_{j}\right|^{2 b_{j}}\left(a_{j}+2 b_{j}\right) r_{j}^{2 b_{j}}+a_{j} t\right\}, \\
\alpha_{j, j+1} & =r_{j}^{a_{j}}\left\{(1-t)\left|w_{j}\right|^{2 b_{j}} r_{j}^{2 b_{j}}+t\right\}, \\
\beta_{j} & =\left\{(1-t)\left|w_{j}\right|^{2 b_{j}}+t\right\}
\end{aligned}
$$

for $j=1, \ldots, n$. The above equality can be written as

$$
\begin{aligned}
A & \left(\begin{array}{c}
\frac{d r_{1}}{d s} \\
\vdots \\
\vdots \\
\vdots \\
\frac{d r_{n}}{d s}
\end{array}\right)=\left(\begin{array}{c}
\beta_{1} \\
\vdots \\
\vdots \\
\vdots \\
\beta_{n}
\end{array}\right) \\
A:= & \left(\begin{array}{ccccc}
\alpha_{1,1} & \alpha_{1,2} & 0 & \ldots & 0 \\
0 & \alpha_{2,2} & \alpha_{2,3} & \ddots & \vdots \\
\vdots & \ddots & \ddots & \ddots & \vdots \\
0 & \ldots & 0 & \alpha_{n-1, n-1} & \alpha_{n-1, n} \\
\alpha_{n, 1} & 0 & \ldots & 0 & \alpha_{n, n}
\end{array}\right) .
\end{aligned}
$$

Observe that the above equality says $d r_{j} / d s(s)$ is independent of $s$. By Lemma 1 , the determinant of $A$ is positive. We first consider the differential $d r_{1} / d s$ and will show that $d r_{1} / d s(0) \geq 0$. Put $m=[n / 2]$, the largest integer such that $m \leq n / 2$. By the Cramer's 
formula, the differential $d r_{1} / d s$ of $r_{1}$ is equal to

$$
\begin{aligned}
\frac{d r_{1}}{d s}= & \frac{1}{\operatorname{det} A} \operatorname{det}\left(\begin{array}{ccccc}
\beta_{1} & \alpha_{1,2} & 0 & \ldots & 0 \\
\vdots & \alpha_{2,2} & \alpha_{2,3} & \ddots & \vdots \\
\vdots & 0 & \ddots & \ddots & \vdots \\
\vdots & \vdots & \ddots & \ddots & \alpha_{n-1, n} \\
\beta_{n} & 0 & \ldots & 0 & \alpha_{n, n}
\end{array}\right) \\
= & \frac{1}{\operatorname{det} A} \sum_{j=1}^{n}(-1)^{j-1} A_{j-1} \beta_{j} A_{j+1}^{\prime} \\
= & \left\{\begin{array}{l}
\frac{1}{\operatorname{det} A} \sum_{k=1}^{m}\left(A_{2 k-2} \beta_{2 k-1} A_{2 k}^{\prime}-A_{2 k-1} \beta_{2 k} A_{2 k+1}^{\prime}\right), \quad n=2 m \\
\frac{1}{\operatorname{det} A} \sum_{k=1}^{m}\left(A_{2 k-2} \beta_{2 k-1} A_{2 k}^{\prime}-A_{2 k-1} \beta_{2 k} A_{2 k+1}^{\prime}\right)+A_{n-1} \beta_{n} A_{n+1}^{\prime}, \quad n=2 m+1
\end{array}\right.
\end{aligned}
$$

where

$$
A_{j-1}=\left\{\begin{array}{ll}
1 & j=1 \\
\prod_{\ell=1}^{j-1} \alpha_{\ell, \ell+1} & j \geq 2
\end{array}, \quad A_{j+1}^{\prime}= \begin{cases}\prod_{\ell=j}^{n-1} \alpha_{\ell+1, \ell+1} & j \leq n-1 \\
1 & j=n\end{cases}\right.
$$

We have

$$
A_{2 k-2} \beta_{2 k-1} A_{2 k}^{\prime}-A_{2 k-1} \beta_{2 k} A_{2 k+1}^{\prime}=A_{2 k-2} A_{2 k+1}^{\prime}\left(\beta_{2 k-1} \alpha_{2 k, 2 k}-\alpha_{2 k-1,2 k} \beta_{2 k}\right) .
$$

As $\alpha_{j, j+1}(1, \ldots, 1)=\beta_{j}$ for $j=1, \ldots, n$, we observe that

$$
\begin{aligned}
& \beta_{2 k-1} \alpha_{2 k, 2 k}(1, \ldots, 1)-\alpha_{2 k-1,2 k}(1, \ldots, 1) \beta_{2 k} \\
= & \beta_{2 k-1}\left\{\alpha_{2 k, 2 k}(1, \ldots, 1)-\beta_{2 k}\right\} \\
= & \beta_{2 k-1}\left\{(1-t)\left|w_{2 k}\right|^{2 b_{2 k}}\left(a_{2 k}+2 b_{2 k}\right)+a_{2 k} t-(1-t)\left|w_{2 k}\right|^{2 b_{2 k}}-t\right\} \\
= & \beta_{2 k-1}\left\{(1-t)\left|w_{2 k}\right|^{2 b_{2 k}}\left(a_{2 k}+2 b_{2 k}-1\right)+\left(a_{2 k}-1\right) t\right\} \geq 0 .
\end{aligned}
$$

The equality holds only if $a_{2 k}=1$ and $b_{2 k}=0$. Note that $w_{i} \neq 0$ for any $i=1, \ldots, n$ by the assumption. Anyway we have

$$
\frac{d r_{1}}{d s}(0) \geq 0
$$

If $n$ is an odd integer, we see that $d r_{1} / d s(0)>0$ by the last unpaired term: $d r_{1} / d s(0) \geq$ $A_{n-1} \beta_{n} A_{n+1}^{\prime}>0$. If there exists some $k$ such that $a_{2 k} \geq 2$, we have also the strict inequality: $d r_{1} / d s(0)>0$.

Next we consider $d r_{k} / d s$ for $k \geq 2$. First observe that our polynomial $f_{I I, t}$ has a symmetry for the cyclic permutation of the coordinates $\sigma=(1,2, \ldots, n)$. Secondly after cyclic change of coordinates, say $\boldsymbol{z}^{\prime}=\left(z_{1}^{\prime}, \ldots, z_{n}^{\prime}\right)=\left(z_{\sigma^{i}(1)}, \ldots, z_{\sigma^{i}(n)}\right)$, the equality $(3)$ does not change. That is, $\boldsymbol{w}^{\prime}(s)=\left(r_{\sigma^{i}(1)} w_{\sigma^{i}(1)}, \ldots, r_{\sigma^{i}(n)} w_{\sigma^{i}(n)}\right)$ is the obtained solution 
curve. The tangent vector $\boldsymbol{v}^{\prime}=d \boldsymbol{w}^{\prime} / d s(0)$ is also equal to $\boldsymbol{v}$ after the corresponding cyclic permutation of coordinates. Therefore we can apply the above argument to have the inequality $\left(d r_{\sigma^{i}(1)} / d s\right)(0) \geq 0$ for any $i$. As we have some $j$ with $a_{j} \geq 2$, this implies

$$
\frac{d r_{j-1}}{d s}(0)>0
$$

Now we are ready to show that $\boldsymbol{v} \neq 0$ and $\boldsymbol{v} \notin T_{\boldsymbol{w}} S_{\varepsilon_{0}}^{2 n-1}$. By the assumption of $\boldsymbol{w}$, the path $\boldsymbol{w}(s)$ satisfies

$$
\begin{aligned}
f_{I I, t}(\boldsymbol{w}(s), \overline{\boldsymbol{w}}(s)) & =(s+1) f_{I I, t}(\boldsymbol{w}, \overline{\boldsymbol{w}}) \equiv 0, \\
\left.\frac{d\|\boldsymbol{w}(s)\|^{2}}{d s}\right|_{s=0} & =2 \sum_{j=1}^{n} r_{j}(0) \frac{d r_{j}}{d s}(0)\left|w_{j}\right|^{2}=2 \sum_{j=1}^{n} \frac{d r_{j}}{d s}(0)\left|w_{j}\right|^{2}>0 .
\end{aligned}
$$

This implies that $\boldsymbol{v} \neq 0$ and $\boldsymbol{v} \notin T_{\boldsymbol{w}} S_{\varepsilon_{0}}^{2 n-1}$.

Case 2: Now we consider the case $I_{\boldsymbol{w}} \neq \emptyset$. Put $I_{\boldsymbol{w}}^{c}$ be the complement of $I_{\boldsymbol{w}}$ and $\mathbb{C}^{* I_{\boldsymbol{w}}^{c}}=$ $\left\{\boldsymbol{z} \in \mathbb{C}^{n} \mid z_{i}=0, i \in I_{\boldsymbol{w}}\right\}$. We consider the mixed polynomial $f^{\prime}(\boldsymbol{z}, \overline{\boldsymbol{z}})=f_{I I, t,\left.\right|_{\mathbb{C}^{* I} I_{\boldsymbol{w}}^{c}} \text {. Let }}$ $J$ be the set of indices $j$ for which $z_{j}$ or $\bar{z}_{j}$ appears in $f^{\prime}$. Note that $J \subset I_{\boldsymbol{w}}^{c}$ but it can be a proper subset.

Case 2-1. Assume that $f^{\prime} \equiv 0$, i.e., $J=\emptyset$. We take simply a real analytic path as follows:

$$
\boldsymbol{w}(s)=(s+1) \boldsymbol{w}
$$

for $s \in \mathbb{R}$. Since $\boldsymbol{w} \in V_{t} \backslash\{O\}$, we observe that

$$
f_{I I, t}(\boldsymbol{w}(s), \overline{\boldsymbol{w}}(s)) \equiv 0,\left.\quad \frac{d\|\boldsymbol{w}(s)\|^{2}}{d s}\right|_{s=0}=2\|\boldsymbol{w}\|^{2}>0 .
$$

Case 2-2. Assume that $f^{\prime} \not \equiv 0$. Then using the connected components of the graph of $f^{\prime}$, we can express $f^{\prime}$ uniquely as follows.

$$
f^{\prime}(\boldsymbol{z}, \overline{\boldsymbol{z}})=f_{1}\left(\boldsymbol{z}_{I_{1}}\right)+\cdots+f_{k}\left(\boldsymbol{z}_{I_{k}}\right)
$$

where the graph of $f_{i}$ is a bamboo and the variables of $f_{i}, f_{j}, i \neq j$ are disjoint and the above expression is a join type expression. Here $I_{i}$ be the set of indices of variables of $f_{i}$ and $\boldsymbol{z}_{I_{i}}=\left(z_{j}\right)_{j \in I_{i}}$ are the variables of $f_{i}$ for $i=1, \ldots, k$. We have the equality $\cup_{i=1}^{k} I_{i}=J$ and $I_{i} \cap I_{j}=\emptyset$ for $i \neq j$. Put $\mathbb{C}^{I_{i}}=\left\{\boldsymbol{z} \in \mathbb{C}^{n} \mid z_{j}=0, j \notin I_{i}\right\}$. Fixing $i$, we will construct a curve $\boldsymbol{w}_{I_{i}}(s)$ on $\mathbb{C}^{* I_{i}}$ so that

$$
f_{i}\left(\boldsymbol{w}_{I_{i}}(s)\right)=(s+1) f_{i}\left(\boldsymbol{w}_{I_{i}}\right) .
$$

The construction of the curve $\boldsymbol{w}_{I_{i}}(s)$ can be reduced to the argument of $[\mathbf{4}$, Lemma 10]. We will give briefly the proof which is based on the argument of [4].

For $j \notin J$, we put $w_{j}(s)=w_{j}$ and $\boldsymbol{w}_{J^{c}}(s)=\boldsymbol{w}_{J^{c}} \in \mathbb{C}^{J^{c}}$ where $\mathbb{C}^{J^{c}}=\left\{\boldsymbol{z} \in \mathbb{C}^{n}\right.$ $\left.z_{j^{\prime}}=0, j^{\prime} \in J\right\}$. Here $\boldsymbol{w}_{J^{c}}$ is the projection of $\boldsymbol{w}$ to $\mathbb{C}^{J^{c}}$. For each $i=1, \ldots, k$, we will construct a curve $\boldsymbol{w}_{I_{i}}(s)$ on $\mathbb{C}^{I_{i}}$ and define $\boldsymbol{w}_{J}(s)=\boldsymbol{w}_{I_{1}}(s)+\cdots+\boldsymbol{w}_{I_{k}}(s)$. Finally we 
define a curve $\boldsymbol{w}(s)=\boldsymbol{w}_{J^{c}}+\boldsymbol{w}_{J}(s) \in \mathbb{C}^{n}$ so that

$$
\begin{aligned}
f_{i}\left(\boldsymbol{w}_{I_{i}}(s)\right) & =(s+1) f_{i}\left(\boldsymbol{w}_{I_{i}}\right), \\
f_{I I, t}(\boldsymbol{w}(s)) & =f^{\prime}(\boldsymbol{w}(s)) \\
& =f_{1}\left(\boldsymbol{w}_{I_{1}}(s)\right)+\cdots+f_{k}\left(\boldsymbol{w}_{I_{k}}(s)\right) \\
& =(s+1)\left\{f_{1}\left(\boldsymbol{w}_{I_{1}}\right)+\cdots+f_{k}\left(\boldsymbol{w}_{I_{k}}\right)\right\} \\
& =(s+1) f_{I I, t}(\boldsymbol{w}) \equiv 0 .
\end{aligned}
$$

So we fix $i$. For simplicity's sake, we assume $I_{i}=\left\{j \mid v_{i} \leq j \leq \tau_{i}\right\}$ with $\tau_{i} \leq n$. The last assumption $\tau_{i} \leq n$ is for the simplicity of the indices. This implies that

$$
f_{i}\left(\boldsymbol{z}_{I_{i}}\right)=\sum_{j=v_{i}}^{\tau_{i}-1} z_{j}^{a_{j}} z_{j+1}\left\{\left|z_{j}\right|^{2 b_{j}}(1-t)+t\right\} .
$$

We will show that there exists a differentiable positive real-valued function solution $\left(r_{v_{i}}(s), \ldots, r_{\tau_{i}}(s)\right)$ of the following equation so that $w_{j}(s)=r_{j}(s) w_{j}, j \in I_{i}$ and

$$
w_{j}^{a_{j}}(s) w_{j+1}(s)\left\{\left|w_{j}(s)\right|^{2 b_{j}}(1-t)+t\right\}=(s+1) w_{j}^{a_{j}} w_{j+1}\left\{\left|w_{j}\right|^{2 b_{j}}(1-t)+t\right\}
$$

for $j=v_{i}, \ldots, \tau_{i}-1$. We first consider the equality

$$
\begin{aligned}
\left(E_{j}^{\prime \prime}\right): \quad r_{j}^{a_{j}}\left\{\left|w_{j}\right|^{2 b_{j}} r_{j}^{2 b_{j}}(1-t)+t\right\} & =s_{j}\left\{\left|w_{j}\right|^{2 b_{j}}(1-t)+t\right\} \\
\text { where } s_{j} & :=(s+1) / r_{j+1}, \quad v_{i} \leq j \leq \tau_{i}-1 .
\end{aligned}
$$

First we define $r_{\tau_{i}}=1$ to start with. The left side of $\left(E_{j}^{\prime \prime}\right)$ is a monotone increasing function of $r_{j}>0$. Thus assuming $s_{j}>0$ and considering $s_{j}$ as an independent variable, we can solve $\left(E_{j}^{\prime \prime}\right)$ in $r_{j}$ as a function of $s_{j}$. Thus we put $r_{j}=\psi_{j}\left(s_{j}\right)$. We claim

Assertion 3.

$$
\begin{gathered}
\psi_{j}(1)=1, \quad \frac{d \psi_{j}}{d s_{j}}\left(s_{j}\right)>0, \\
\psi_{j}\left(s_{j}\right)^{a_{j}} \leq s_{j}, j=v_{i}, \ldots, \tau_{i}-1 .
\end{gathered}
$$

Proof. For $j=\tau_{i}-1$, the assertion is obvious. Assume that $j<\tau_{i}-1$. The assertion (6) is obvious. The assertion (7) follows from (6), as $\psi\left(s_{j}\right)$ is monotone increasing on $s_{j}$ and

$$
\left|w_{j}\right|^{2 b_{j}} r_{j}^{2 b_{j}}(1-t)+t \geq\left|w_{j}\right|^{2 b_{j}}(1-t)+t, r_{j} \geq 1
$$

Now we define $s_{j}(s)$ and $r_{j}(s)$ inductively from $j=\tau_{i}$ downward (more precisely from the right end vertex of the graph to the left) as follows:

$$
r_{\mu_{i}}(s)=1, \quad s_{j}(s)=(s+1) / r_{j+1}(s), r_{j}(s)=\psi_{j}\left(s_{j}(s)\right)
$$

for $v_{i} \leq j \leq \tau_{i}-1$. 
Assertion $4 . \quad s_{j}(s) \geq 1$ and $r_{j}(s) \geq 1$ for $j=v_{i}, \ldots, \tau_{i}-1$ and $s \geq 0$.

Proof. We show the assertion by a downward induction. For $j=\tau_{i}-1$, the assertion is obvious. By the inequality (7), we have for $j<\tau_{i}-1$

$$
\begin{aligned}
s_{j}(s)^{a_{j+1}} & =\left(\frac{s+1}{r_{j+1}(s)}\right)^{a_{j+1}}=\frac{(s+1)^{a_{j+1}}}{\psi_{j+1}\left(s_{j+1}(s)\right)^{a_{j}+1}} \\
& \geq \frac{(s+1)^{a_{j+1}}}{s_{j+1}(s)}=(s+1)^{a_{j+1}-1} r_{j+2}(s)
\end{aligned}
$$

for $s \geq 0$. By the definition of $r_{j}(s)$ and Assertion $3, s_{j}(s) \geq 1$ and $r_{j}(s) \geq 1$ for $j=v_{i}, \ldots, \tau_{i}-1$ and $s \geq 0$.

By Assertion 3 and Assertion 4, we see easily that

$$
\left\{\begin{array}{l}
\frac{d r_{\tau_{i}-1}}{d s}(0)>0 \\
\frac{d r_{j}}{d s}(0) \geq 0, j=v_{i}, \ldots, \tau_{i}-2 .
\end{array}\right.
$$

Now we define the curve $\boldsymbol{w}_{I_{i}}(s)$ on $\mathbb{C}^{I_{i}}$ by

$$
w_{j}(s)=r_{j}(s) w_{j}, \quad j \in I_{i} .
$$

As a vector in $\mathbb{C}^{n}$, the other coefficients of $\boldsymbol{w}_{I_{i}}(s)$ are defined to be zero. Then by the construction we have

$$
\begin{array}{r}
\boldsymbol{w}_{I_{i}}(0)=\boldsymbol{w}_{I_{i}}, \quad f_{i}\left(\boldsymbol{w}_{I_{i}}(s), \overline{\boldsymbol{w}}_{I_{i}}(s)\right)=(s+1) f_{i}\left(\boldsymbol{w}_{I_{i}}, \overline{\boldsymbol{w}}_{I_{i}}\right), \\
\left.\frac{d\left\|\boldsymbol{w}_{I_{i}}\right\|^{2}}{d s}\right|_{s=0} \geq 2 \frac{d r_{\tau_{i}-1}}{d s}(0)\left|w_{\tau_{i}-1}\right|^{2}>0
\end{array}
$$

where $|s| \ll 1$ and $1 \leq i \leq k$. After constructing $\boldsymbol{w}_{I_{i}}(s)$ for each $i=1, \ldots, k$, we define a smooth curve $\boldsymbol{w}(s)=\left(w_{1}(s), \ldots, w_{n}(s)\right)$ by the summation

$$
\begin{aligned}
\boldsymbol{w}(s) & =\boldsymbol{w}_{J^{c}}(s)+\boldsymbol{w}_{J}(s), \\
\boldsymbol{w}_{J}(s) & =\boldsymbol{w}_{I_{1}}(s)+\cdots+\boldsymbol{w}_{I_{k}}(s) .
\end{aligned}
$$

Then $\boldsymbol{w}(s)$ satisfies

$$
\begin{aligned}
f_{I I, t}(\boldsymbol{w}(s)) & =f^{\prime}(\boldsymbol{w}(s), \overline{\boldsymbol{w}}(s))=\sum_{i=1}^{k} f_{i}\left(\boldsymbol{w}_{I_{i}}(s), \overline{\boldsymbol{w}}_{I_{i}}(s)\right) \\
& =(s+1) \sum_{i=1}^{k} f_{i}\left(\boldsymbol{w}_{I_{i}}, \overline{\boldsymbol{w}}_{I_{i}}\right)=(s+1) f^{\prime}(\boldsymbol{w}, \overline{\boldsymbol{w}}) \\
& =(s+1) f_{I I, t}(\boldsymbol{w}, \overline{\boldsymbol{w}}) \equiv 0, \\
\left.\frac{d\|\boldsymbol{w}(s)\|^{2}}{d s}\right|_{s=0} & =\left.\sum_{i=1}^{k} \frac{d\left\|\boldsymbol{w}_{I_{i}}(s)\right\|^{2}}{d s}\right|_{s=0}>0 .
\end{aligned}
$$


Thus defining $\boldsymbol{v}:=d \boldsymbol{w} / d s(0)$, we conclude $\boldsymbol{v} \in T_{\boldsymbol{w}} V_{t} \backslash T_{\boldsymbol{w}} S_{\varepsilon_{0}}^{2 n-1}$. This completes the proof of the transversality.

REMARK 1. In the above argument, if $v_{n}$ is a vertex of the graph of $f_{i}$ and it is not the right end vertex, we use the expression $I_{i}=\left\{j \bmod n \mid v_{i} \leq j \leq \tau_{i}\right\}$ with $\tau_{i}>n$. This implies that

$$
f_{i}\left(\boldsymbol{z}_{I_{i}}\right)=\sum_{j=v_{i}}^{\tau_{i}-1} z_{j}^{a_{j}} z_{j+1}\left\{\left|z_{j}\right|^{2 b_{j}}(1-t)+t\right\}
$$

where $z_{j+n}=z_{j}, a_{j+n}=a_{j}, b_{j+n}=b_{j}$. We do the same argument as above starting the right end variable $z_{\tau_{i}}=z_{\tau_{i}-n}$.

\subsection{Applications.}

Corollary 1. Let $V_{t}$ be the hypersurface defined by $f_{I I, t}$ and let $K_{t, \varepsilon}$ be its link. Then there exists an isotopy $\psi_{t}:\left(S_{\varepsilon}^{2 n-1}, K_{0, \varepsilon}\right) \rightarrow\left(S_{\varepsilon}^{2 n-1}, K_{t, \varepsilon}\right)$ for $0 \leq t \leq 1$ with $\psi_{0}=\mathrm{id}$.

This is immediate from Ehresmann's fibration theorem $([\mathbf{9}])$. As for the Milnor fibration of the second type, we have:

Corollary 2. For a fixed $\varepsilon>0$, there exists a positive real number $\eta_{0}$ so that $f_{I I, t}^{-1}(\eta)$ and $S_{\varepsilon}^{2 n-1}$ intersect transversely for any $\eta,|\eta| \leq \eta_{0}$ and $0 \leq t \leq 1$. In particular this implies that there exists a family of diffeomorphisms $\psi_{t}: \partial E_{0}\left(\eta_{0}, \varepsilon\right) \rightarrow \partial E_{t}\left(\eta_{0}, \varepsilon\right)$ such that the following diagram is commutative:

$$
\begin{array}{ccc}
\partial E_{0}\left(\eta_{0}, \varepsilon\right) & \stackrel{\psi_{t}}{\longrightarrow} \partial E_{t}\left(\eta_{0}, \varepsilon\right) \\
\downarrow_{\mid}^{\mid} f_{I I, 0} & & \rfloor_{\downarrow}^{\mid} f_{I I, t} \\
S_{\eta_{0}}^{1} & = & S_{\eta_{0}}^{1}
\end{array}
$$

where $\partial E_{t}\left(\eta_{0}, \varepsilon\right)=\left\{\boldsymbol{z} \in \mathbb{C}^{n}|| f_{I I, t}(\boldsymbol{z}) \mid=\eta_{0},\|\boldsymbol{z}\| \leq \varepsilon\right\}$.

Proof. Fix a positive real number $\varepsilon$. Let

$$
\begin{aligned}
\partial \mathcal{E}\left(\eta_{0}, \varepsilon\right) & :=\left\{(\boldsymbol{z}, t) \in \mathbb{C}^{n} \times[0,1]|| f_{I I, t}(\boldsymbol{z}) \mid=\eta_{0},\|\boldsymbol{z}\| \leq \varepsilon\right\} \\
\partial^{2} \mathcal{E}\left(\eta_{0}, \varepsilon\right) & :=\left\{(\boldsymbol{z}, t) \in \mathbb{C}^{n} \times[0,1]|| f_{I I, t}(\boldsymbol{z}) \mid=\eta_{0},\|\boldsymbol{z}\|=\varepsilon\right\} .
\end{aligned}
$$

Since $S_{\varepsilon}^{2 n-1}$ intersects with $V_{t}$ transversely and $S_{\varepsilon}^{2 n-1} \cap V_{t}$ is compact for any $0 \leq t \leq 1$, there exists a positive real number $\eta_{0}$ such that $f_{I I, t}^{-1}(\eta)$ and $S_{\varepsilon}^{2 n-1}$ intersect transversely for any $\eta,|\eta| \leq \eta_{0}$ and $0 \leq t \leq 1$. Thus the projection $\pi^{\prime}:\left(\partial \mathcal{E}\left(\eta_{0}, \varepsilon\right), \partial^{2} \mathcal{E}\left(\eta_{0}, \varepsilon\right)\right) \rightarrow$ $[0,1]$ is a proper submersion. By the Ehresmann's fibration theorem $[\mathbf{9}], \pi^{\prime}$ is a locally trivial fibration over $[0,1]$. So the projection $\pi^{\prime}$ induces a family of isomorphisms $\psi_{t}$ : $\partial E_{0}\left(\eta_{0}, \varepsilon\right) \rightarrow \partial E_{t}\left(\eta_{0}, \varepsilon\right)$ of fibrations for any $\boldsymbol{z}$ with $\left|f_{I I, t}(\boldsymbol{z})\right| \leq \eta_{0}$ and $0 \leq t \leq 1$.

Now, we consider again the Milnor fibration of the link complement. Consider the mapping 


$$
f_{I I, t} /\left|f_{I I, t}\right|: S_{\varepsilon}^{2 n-1} \backslash K_{t, \varepsilon} \rightarrow S^{1} .
$$

As $f_{I I, t}(\boldsymbol{z}, \overline{\boldsymbol{z}})$ is polar weighted homogeneous polynomial, the $S^{1}$-action gives nonvanishing vector field, denoted as $\partial / \partial \theta$ on $S_{\varepsilon}^{2 n-1} \backslash K_{t, \varepsilon}$ so that $f_{I I, t}(c \circ \boldsymbol{z})=c^{d_{p}} f_{I I, t}(\boldsymbol{z})$ for $c \in S^{1}$, this gives a fibration structure for (8) for any $\varepsilon>0$ and we call it a spherical Milnor fibration or a Milnor fibration of the first description. The isomorphism class of the fibration does not depend on $\varepsilon$. Consider two fibrations

$$
f_{I I, t}: \partial E_{t}\left(\eta_{0}, \varepsilon\right) \rightarrow S_{\eta_{0}}^{1}, \quad f_{I I, t} /\left|f_{I I, t}\right|: S_{\varepsilon}^{2 n-1} \backslash K_{t, \varepsilon} \rightarrow S^{1} .
$$

The first fibration is called a Milnor fibration of the second description or a tubular Milnor fibration. The isomorphism class of the tubular fibration does not depend on the choice of $\varepsilon$ and $\eta_{0} \ll \varepsilon$. As we know that two fibrations are isomorphic for sufficiently small $\varepsilon>0$ and any $t([\mathbf{3}$, Theorem 36$])$, they are isomorphic for any $\varepsilon$. Combining this and Corollary 2, we can sharpen Corollary 1 as follows.

Corollary 3. Let $\psi_{t}:\left(S_{\varepsilon}^{2 n-1}, K_{0, \varepsilon}\right) \rightarrow\left(S_{\varepsilon}^{2 n-1}, K_{t, \varepsilon}\right)$ be an isotopy in Corollary 1. $\psi_{t}$ can be constructed so that the following diagram is commutative.

$$
\begin{aligned}
& S_{\varepsilon}^{2 n-1} \backslash K_{0, \varepsilon} \stackrel{\psi_{t}}{\longrightarrow} S_{\varepsilon}^{2 n-1} \backslash K_{t, \varepsilon} \\
& \underset{S^{1}}{\mid \underset{I I, 0}{\mid} \underset{i d}{\longrightarrow}} \underset{S^{1}}{\longrightarrow} f_{I I, 0}\left|f_{I I, t} /\right| f_{I I, t} \mid
\end{aligned}
$$

Taking $t=1$, we get a positive answer to the conjecture in [4].

Proof. Choose a positive real number $\eta_{0}$ as in Corollary 2. Consider the cobordism variety $\mathcal{V}_{\varepsilon}:=\left\{(\boldsymbol{z}, t) \in S_{\varepsilon}^{2 n-1} \times[0,1] \mid f_{I I, t}(\boldsymbol{z}, \overline{\boldsymbol{z}})=0\right\}$ and its open neighborhood $\mathcal{W}_{\eta}:=\left\{(\boldsymbol{z}, t) \in S_{\varepsilon}^{2 n-1} \times[0,1]|| f_{I I, t}(\boldsymbol{z}) \mid<\eta\right\}$ of $\mathcal{V}_{\varepsilon}$. Consider the projection mapping

$$
\pi: S_{\varepsilon}^{2 n-1} \times[0,1] \rightarrow[0,1], \quad(\boldsymbol{z}, t) \mapsto t .
$$

Let $(\partial / \partial \theta)^{\prime}$ be the projection of the gradient vector of $\mathfrak{I} \log f_{I I, t}(\boldsymbol{z}, \overline{\boldsymbol{z}})$ to the tangent space of $S_{\varepsilon}^{2 n-1} \times[0,1] \backslash \mathcal{V}_{\varepsilon}$. Using the vector field $\partial / \partial \theta$ on $S_{\varepsilon}^{2 n-1} \times[0,1]$, we see easily that $(\partial / \partial \theta)^{\prime}$ is a non-vanishing vector on $S_{\varepsilon}^{2 n-1} \times[0,1] \backslash \mathcal{V}_{\varepsilon}$ which is linearly independent with $\partial / \partial t$ over $\mathbb{R}$. Now we construct a vector filed $\mathcal{X}$ on $S_{\varepsilon}^{2 n-1} \times[0,1] \backslash \mathcal{V}_{\varepsilon}$ such that

1. $d \pi_{*}(\mathcal{X}(\boldsymbol{z}, t))=\partial / \partial u$ and $\left\{\mathcal{X}(\boldsymbol{z}, t),(\partial / \partial \theta)^{\prime}(\boldsymbol{z}, t)\right\}$ are orthogonal.

2. For $(\boldsymbol{z}, t) \in \mathcal{W}_{\eta_{0} / 2},\left\{\mathcal{X}(\boldsymbol{z}, t)\right.$, grad $\left.\left|f_{I I, t}\right|(\boldsymbol{z}, t)\right\}$ are also orthogonal.

Here $\partial / \partial u$ is a tangent vector on $[0,1]$. The condition (1) implies the argument of $f_{I I, t}$ does not change along the integral curve of $\mathcal{X}$. The conditions (1) and (2) implies the integral curve of $\mathcal{X}$ keeps the level $f_{I I, t}=\eta$ for any $\eta$ with $|\eta| \leq \eta_{0} / 2$. Thus integral curves of vector field $\mathcal{X}$ exists over $[0,1]$ and we construct the isotopy $\psi_{t}$ using the integration curves of $\mathcal{X}$.

REMARK 2. Let $f(\boldsymbol{z}, \overline{\boldsymbol{z}})=\sum_{i=1}^{m} c_{i} \boldsymbol{z}^{\nu_{i}} \overline{\boldsymbol{z}}^{\mu_{i}}$ be a full simplicial mixed polynomial and $g(\boldsymbol{z})$ be the associated Laurent polynomial of $f$. The last author defined a canonical diffeomorphism of $\varphi: \mathbb{C}^{* n} \rightarrow \mathbb{C}^{* n}$ as follows $([\mathbf{2}])$ : 


$$
\begin{aligned}
\varphi: \mathbb{C}^{* n} & \rightarrow \mathbb{C}^{* n}, \\
\boldsymbol{z}=\left(\rho_{1} \exp \left(i \theta_{1}\right), \ldots, \rho_{n} \exp \left(i \theta_{n}\right)\right) & \mapsto \boldsymbol{w}=\left(\xi_{1} \exp \left(i \theta_{1}\right), \ldots, \xi_{n} \exp \left(i \theta_{n}\right)\right)
\end{aligned}
$$

where $\left(\rho_{1}, \ldots, \rho_{n}\right)$ and $\left(\xi_{1}, \ldots, \xi_{n}\right)$ satisfy

$$
(N+M)\left(\begin{array}{c}
\log \rho_{1} \\
\vdots \\
\log \rho_{n}
\end{array}\right)=(N-M)\left(\begin{array}{c}
\log \xi_{1} \\
\vdots \\
\log \xi_{n}
\end{array}\right)
$$

where $N=\left(\nu_{1}, \ldots, \nu_{n}\right)$ and $M=\left(\mu_{1}, \ldots, \mu_{n}\right)$. Then $\varphi$ satisfies that $\varphi\left(\mathbb{C}^{* n} \cap f^{-1}(c)\right)=$ $\mathbb{C}^{* n} \cap g^{-1}(c)$ for any $c \in \mathbb{C}([\mathbf{2}$, Theorem 10]). However $\varphi$ cannot be extended to a homeomorphism of $\mathbb{C}^{n} \backslash\{O\}$ to itself in general, except the case of mixed Brieskorn polynomial.

ExAmple 1. We will give an example of the above remark. Let $f(\boldsymbol{z}, \overline{\boldsymbol{z}})$ be a simplicial polynomial defined by

$$
f(\boldsymbol{z}, \overline{\boldsymbol{z}})=z_{1}^{3} \bar{z}_{1} z_{2}+z_{2}^{3} \bar{z}_{2} z_{3}+z_{3}^{3} \bar{z}_{3} z_{1} .
$$

Then the diffeomorphism of $\varphi: \mathbb{C}^{* 3} \rightarrow \mathbb{C}^{* 3}, \boldsymbol{z}=\left(z_{1}, z_{2}, z_{3}\right) \mapsto \boldsymbol{w}=\left(w_{1}, w_{2}, w_{3}\right)$ is given by

$$
\left(\begin{array}{l}
w_{1} \\
w_{2} \\
w_{3}
\end{array}\right)=\left(\begin{array}{l}
\left|z_{1}\right|^{17 / 9}\left|z_{2}\right|^{-4 / 9}\left|z_{3}\right|^{2 / 9} \exp \left(i \theta_{1}\right) \\
\left|z_{1}\right|^{2 / 9}\left|z_{2}\right|^{17 / 9}\left|z_{3}\right|^{-4 / 9} \exp \left(i \theta_{2}\right) \\
\left|z_{1}\right|^{-4 / 9}\left|z_{2}\right|^{2 / 9}\left|z_{3}\right|^{17 / 9} \exp \left(i \theta_{3}\right)
\end{array}\right)
$$

The above map cannot extend to a continuous map on the coordinate planes $\left\{\left(z_{1}, z_{2}, z_{3}\right) \in \mathbb{C}^{3} \mid z_{1} z_{2} z_{3}=0\right\}$ as the negative exponents in the above description. So the map $\varphi$ cannot extend to a homeomorphism of $\mathbb{C}^{3} \backslash\{O\}$ to itself.

\section{References}

[1] J. L. Cisneros-Molina, Join theorem for polar weighted homogeneous singularities, Singularities II, edited by J. P. Brasselet, J. L. Cisneros-Molina, D. Massey, J. Seade and B. Teissier, Contemp, Math., 475, Amer. Math. Soc., Providence, RI, 2008, 43-59.

[2] M. Oka, Topology of polar weighted homogeneous hypersurfaces, Kodai Math. J., 31 (2008), 163-182.

[ 3 ] M. Oka, Non-degenerate mixed functions, Kodai Math. J., 33 (2010), 1-62.

[ 4 ] M. Oka, On Mixed Brieskorn variety, Contemp. Math., 538 (2011), 389-399.

[5] P. Orlik and P. Wagreich, Isolated singularities of algebraic surfaces with $\mathbb{C}^{*}$ action, Ann. of Math., 93 (1971), 205-228.

[6] M. A. S. Ruas, J. Seade and A. Verjovsky, On real singularities with a Milnor fibration, Trends Math., (eds. A. Libgober and M. Tibăr), Birkhäuser, Basel, 2003, 191-213.

[ 7 ] J. Seade, Fibered links and a construction of real singularities via complex geometry, Bull. Braz. Math. Soc., 27 (1996), 199-215.

[ 8 ] J. Seade, On the Topology of Isolated Singularities in Analytic Spaces, Progress in Mathematics, 241, Birkhäuser, 2005.

[ 9 ] J. A. Wolf, Differentiable fibre spaces and mappings compatible with Riemannian metrics, Michigan Math. J., 11 (1964), 65-70. 


\section{Kazumasa InABA}

Mathematical Institute

Tohoku University

Sendai 980-8578, Japan

E-mail: sb0d02@math.tohoku.ac.jp

\section{Masayuki KAWASHIMA}

Department of Information Science

Okayama University of Science

1-1 Ridai-cho, Kitaku

Okayama 700-0005, Japan

E-mail: kawashima@mis.ous.ac.jp

\section{Mutsuo OKA}

Department of Mathematics Tokyo University of Science

1-3 Kagurazaka, Shinjuku-ku

Tokyo 162-8601, Japan

E-mail: oka@rs.kagu.tus.ac.jp 